\title{
Estrogen and progesterone receptors in human papilloma virus-related cervical neoplasia
}

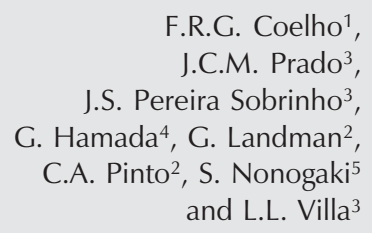

F.R.G. Coelho ${ }^{1}$,

J.C.M. Prado ${ }^{3}$,

J.S. Pereira Sobrinho ${ }^{3}$,

G. Hamada ${ }^{4}$, G. Landman²,

C.A. Pinto ${ }^{2}$, S. Nonogaki ${ }^{5}$ and L.L. Villa ${ }^{3}$

\author{
Departamentos de ${ }^{1}$ Ginecologia and ${ }^{2}$ Patologia, Hospital do Câncer, \\ Fundação Antonio Prudente, São Paulo, SP, Brasil \\ ${ }^{3}$ Instituto Ludwig de Pesquisa sobre o Câncer, São Paulo, SP, Brasil \\ ${ }^{4}$ Centro de Prevenção de Doenças Nikkey, Hospital Santa Cruz, \\ São Paulo, SP, Brasil \\ Instituto Adolfo Lutz, São Paulo, SP, Brasil
}

\section{Correspondence}

F.R.G. Coelho

Rua Décio de Almeida Filho, 421

13084-710 Campinas, SP

Brasil

E-mail: frgcoelho@lexxa.com.br

Research supported by CAPES.

Publication supported by FAPESP.

Received February 19, 2003

Accepted September 18, 2003

\begin{abstract}
Estrogen (ER) and progesterone (PR) receptors in the normal uterine cervix, cervical intraepithelial neoplasia and invasive carcinoma were studied in consecutive samples from Hospital do Câncer, São Paulo, between 1996 and 1997. Tissue was collected by removing a fragment of the tumoral area using a 5-mm diameter biopsy punch, followed by removal of a macroscopically normal area as close as possible from the tumor. Histopathological confirmation was obtained for all specimens analyzed. A total of 24 normal tissues, 17 cases of cervical intraepithelial neoplasia and 7 of invasive carcinomas were studied. The ER/PR ratio was determined by immunohistochemistry using monoclonal antibodies specific for each receptor. Adjacent tissue slides were submitted to generic PCR for human papillomavirus (HPV) DNA detection followed by typing by dot blot hybridization. About half (45.8\%) of the tumors were HPV DNA positive while $29.1 \%$ of the patients were also HPV positive in their respective normal tissue. ER was negative in the tumoral epithelium of $11 \mathrm{HPV}$-positive patients (P $=0.04)$. There was a trend in the ER distribution in normal tissue that was opposite to that from lesions, but it was not statistically significant $(\mathrm{P}=0.069)$. No difference in ER distribution in stromal tissues was observed between HPV-positive and HPV-negative tissues. PR staining was negative in the epithelium of all cases studied. The results obtained from this small number of cases cannot be considered to be conclusive but do suggest that factors related to viral infection affect the expression of these ER/PR cervix receptors.
\end{abstract}

Human papillomaviruses (HPVs) are commonly found in the genital tract of sexually active women and studies have confirmed that persistent infection with highrisk types of HPV in the cervix precedes cervical cancer (1,2). However, although HPV is a necessary cause it cannot be considered
Key words

- Estrogen receptor

- Progesterone receptor

- Cervical cancer

- Cervical intraepithelial neoplasia

- Human papillomavirus sufficient to trigger all the changes required to lead to this neoplasia (3). Mutagenic and immunosuppressive agents can cooperate with HPV through different mechanisms (2). Interaction is known to occur between HPV and some types of hormones, particularly estrogens, and their antagonists, resulting in 
the alteration of viral gene expression (4). It is known that the upstream regulatory region of several HPVs contains glucocorticoidresponsive elements (5). These hormones have a receptor similar to that of the estrogen receptor (ER) hormone (5-7).

A series of reports have shown that mean ER positivity in the cervical mucosa can vary from 13 to $100 \%$. For progesterone receptors $(\mathrm{PR})$ the variation is even wider, i.e., 0 to $100 \%$ of tissues from the uterine cervix have been reported to be PR positive (8-12). No statistically significant correlations have been found for the ER/PR ratio in the cervix when related to menstrual cycle, menopause, histological type or ploidy analysis (8-11).

A 1989 study (13) demonstrated a gradient of ER detection in the uterine cervix: while a low expression of ER was observed in moderate dysplasia, cases of severe dysplasia and cervical cancer were negative for the receptor.

One major explanation for these discrepancies could be related to the location of the receptors, which can be found either in the epithelium or in the stroma, to the size of the tissue analyzed, and to the method for receptor detection $(8,9,13,14)$. In an attempt to clarify some of these issues we sought to evaluate the concentration of ER and PR in samples from the normal uterine cervix, from cervical intraepithelial neoplasia (CIN 1, 2 and 3) and from invasive carcinoma, associated or not with the presence of HPV DNA. Consecutive cases of CIN and invasive carcinomas of the uterine cervix were obtained at the Gynecology Department of the Hospital do Câncer, Fundação Antônio Prudente, São Paulo, Brazil, between May 1996 and March 1997. The following samples were obtained: 5 CIN 1, 3 CIN 2, 9 CIN 3, and 7 cervical invasive carcinomas (4 squamous cell carcinomas and 3 adenocarcinomas). Patient age ranged from 23 to 50 years (mean: 39 years). The following eligibility criteria were established for patient inclusion in the study: premenopausal women who had not used oral contraceptives for the last 2 years and a histologically confirmed diagnosis of CIN and invasive carcinoma, not previously treated. A fragment of tumoral tissue was removed with a 5-mm diameter disposable biopsy punch (Stiefel Company, Coral Gables, FL, USA). This collection device was chosen to reduce the collection of adjacent stromal tissue. Next, we searched for macroscopically normal tissue close to the tumoral area and obtained a second tissue fragment with another biopsy punch to avoid any possible tissue cross-contamination. Each of the tissues was divided into three equal parts, resulting in six fragments. Two of the fragments corresponding to either normal or abnormal tissues were immediately placed in liquid nitrogen, followed by storage at $-70^{\circ} \mathrm{C}$ for variable periods of time before being tested for HPV DNA. The third fragment was immersed in buffered formalin for routine histopathological procedures and immunohistochemical determination of ER and PR. DNA was extracted from the tissue by a routine procedure (Rapid Prep. Genomic Isolation, Pharmacia Biotech, Uppsala, Sweden). DNA amplification was performed using the polymerase chain reaction (PCR) according to previously described protocols employing generic primers MY09/MY11 capable of amplifying a fragment of the L1 gene of all known genital HPVs (15). Reactions were processed in a thermal cycler (Perkin-Elmer Cetus, Foster City, CA, USA). HPV typing was performed by dot blot hybridization as previously described (15). This procedure allows the identification of more than $30 \mathrm{HPV}$ types. In 4 cases, the PCR products were further typed by restriction fragment length polymorphism (16). Positive and negative reaction controls were always used in the PCR assays. As an internal control for DNA quality we amplified a fragment of the $B$-globin gene and the reaction was positive for $100 \%$ of the specimens. The tissues were submitted to standard immuno- 
histochemical evaluation. The monoclonal antibody used for ER was the $6 \mathrm{~F} 11$ clone (NCL-ER - 6F11, Novocastra, UK) diluted $1 / 20$. The monoclonal antibody, clone $1 \mathrm{~A} 6$ (NCL-PGR, Novocastra, UK), also diluted $1 / 20$, was used for PR determination. Positive and negative controls were used in all assays. The percentage of positive cells (nuclear positivity only) was determined in the stroma, as well as in the epithelium, normal mucosa, atypical epithelium (CIN 1, CIN 2 and CIN 3), and invasive carcinoma. A differential cell counter (Diffcount, model MD-8, Modulus Data System, Santa Clara, CA, USA) was used. In all cases, a minimum of 100 and a maximum of 200 cells were counted, and the results are reported as frequency of positive cells.

The chi-square test was used for statistical analysis. Since normal tissue, considered as a control, was removed from the same patient, we also used the McNemar test (17).

Among 24 patients, HPV DNA was detected in $11(45.8 \%)$ cases with cervical lesions (CIN and cancer), while 7 (29\%) samples were positive in the control tissues. HPV16 was the most frequent type, being present in 12 tissue samples (8 CIN and cancer and 4 control tissues), followed by 4 type 58 positive samples ( $2 \mathrm{CIN}$ and their controls), and finally by type 33 , present in one invasive carcinoma and in its respective control. As expected, we observed a gradient of HPV positivity ranging from $29.2 \%$ for normal tissue to $35.3 \%$ for CIN 1, 2, and 3 together and to $71.4 \%$ for invasive carcinomas. These frequencies are somewhat lower than the previous reports in which almost $100 \%$ of the invasive cervical cancers bear HPV-DNA sequences (18). The low HPV positivity observed could be due to the limited amount of tissue available, although the globin control of all specimens was positive. One should also consider that low-grade CINs tend to be heterogeneous and it is possible that the fragment submitted to PCR may not contain HPV-positive cells. Nevertheless, in the present study there was no case in which the tumoral tissue was HPV DNA negative and the adjacent normal tissue was HPV positive; moreover, the same HPV types were found in both tumor and control tissues. This could be explained by the physical proximity of the two biopsies, although extreme care was taken to avoid cross-contamination at the time of sample collection and processing.

The expression of ER and PR was analyzed both in the epithelium and in the stroma of normal and tumoral tissues. We observed that $20.8 \%$ of the tumors ( 5 of 24 cases of CIN and invasive cancer) were positive for ER expression in the epithelium. On the other hand, half of the adjacent normal tissue samples (12 of 24) were positive for ER. However, this difference was not significant, as determined by comparison using the chi-square test $(\mathrm{P}=0.069)$. Furthermore, the distribution of ER in the stroma was identical for normal and tumoral tissues with positivity ranging from 20.8 to $25 \%$ in the tissues studied $(\mathrm{P}=1.000)$. Similar results were obtained for PR present in the stroma of both normal and tumoral tissues. Interestingly, PR expression was not observed in the epithelium of any case studied and all 3 adenocarcinomas studied were both ER and PR negative.

Stratification on the basis of the HPV result revealed that all HPV-positive CIN and invasive cancers were negative for ER expression in the epithelium. Conversely, HPV-negative tumors expressed ER in 38.4\% of cases ( 5 of 13; $\mathrm{P}=0.041$; Table 1). A similar inverse distribution of epithelial ER was observed in normal tissues, but there was no statistically significant difference $(\mathrm{P}$ $=0.371$; Table 2). Expression of ER in stromal cells was similar for HPV-positive and -negative tumors $(\mathrm{P}=1.0)$. On the other hand, an increased number of ER was observed in the stroma of normal tissue, i.e., 5 ER-positive cases of 7 HPV-positive cases (71.4\%). Once more, in HPV-negative nor- 
mal tissues, ER positivity was $41.1 \%$ (Table 2 ). There was no statistically significant difference in the frequency of detection of HPV DNA between tumoral and normal tissues ( $P$ $=0.133$ ).

Studies carried out to assess the presence of ER and PR in the uterine cervix have reported different results. These variations may have resulted from differences between the patients and/or the methodology used for the evaluation of the receptors. We chose an assay based on immunocytochemistry because of its easy application to small tissue fragments, consequently avoiding problems related to the possibility of mixing epithelial and stromal cells. Cellular heterogeneity, not taken into consideration in most studies, may account for the variation in ER/PR concentration in the tumor or normal epithelium or even in the subjacent stroma (13). We sought to control for this variation by sampling both tumoral and normal tissues from

Table 1. Association between human papillomavirus (HPV) and estrogen receptor in the epithelium of cervical intraepithelial neoplasias and invasive cervical cancers.

\begin{tabular}{lrcr}
\hline $\begin{array}{l}\text { Estrogen } \\
\text { receptor }\end{array}$ & Negative & Positive & Total \\
\hline Negative & $8(61.5 \%)$ & $11(100 \%)$ & $19(79.1 \%)$ \\
Positive & $5(38.4 \%)$ & 0 & $5(20.8 \%)$ \\
Total & $13(100 \%)$ & $11(100 \%)$ & $24(100 \%)$ \\
\hline
\end{tabular}

A statistically significant association was detected ( $P=0.041$, chi-square test).

Table 2. Association between human papillomavirus (HPV) and estrogen receptors in the normal epithelium of the uterine cervix.

\begin{tabular}{lrll}
\hline $\begin{array}{l}\text { Estrogen } \\
\text { receptor }\end{array}$ & Negative & Positive & Total \\
\hline Negative & $10(58.8 \%)$ & $2(28.5 \%)$ & $12(50 \%)$ \\
Positive & $7(41.1 \%)$ & $5(71.4 \%)$ & $12(50 \%)$ \\
Total & $17(100 \%)$ & $7(100 \%)$ & $24(100 \%)$ \\
\hline
\end{tabular}

There was no statistically significant association ( $P=0.371$, chi-square test). the same patient at the same time, therefore achieving a very good homogeneity of the samples submitted to comparison. However, one may argue that due to the same embryologic origin and proximity of the tumor, the normal counterpart considered here as control was probably exposed to the same factors that may have caused the lesion. Nevertheless, the differences observed in the expression of these steroid receptors are reassuring.

ER expression in the epithelium was observed in only $20 \%$ of HPV-positive tumors, as compared to $50 \%$ of the normal tissues ( $\mathrm{P}$ $=0.069)$. This result is similar to that obtained in previous studies in which ER expression was found to be inversely associated with disease status $(8,9,12)$. On the other hand, a similar ER distribution was observed in the stroma of both tumors and normal tissues. This trend, although not statistically significant, may be related to the fact that HPV replicates exclusively in the epithelial layer. In fact, the distribution of epithelial ER in our study presented greater negativity in the lesions restricted to the epithelial layer (CIN 1, CIN 2, and CIN 3). The $100 \%$ ER negativity in the epithelium of invasive carcinomas detected in our sample leads us to speculate that the invasive lesions "contaminate" the subjacent stromal tissue, which then will primarily consist of tumoral cells. When the tissue fragment to be analyzed is small, as in our study, this aspect of predominance of tumoral cells in stromal tissue may contribute to ER negativity.

Our negative results concerning PR expression in the epithelium of both tumoral and normal tissues contrast with previous reports (10-12). We have excluded a technical problem, since the stroma of the same cases reacted positively. Recent studies using an enzyme-linked immunoassay have shown a 74 to $100 \%$ variation in PR positivity in the normal uterine cervix (10-12). Moreover, PR is expressed at high levels in low and high grade CIN lesions, whereas 
very low levels of this receptor are observed in invasive cervical carcinomas (8). Since $\mathrm{PR}$ assembly is an estrogen-dependent process, the presence of functional ER can be considered to be necessary for the formation of PR (9). In our study, all HPV-positive tumors were negative for epithelial expression of ER, while about $38 \%$ of the HPVnegative cases were ER positive $(P=0.041)$.

The transcriptional activation of the human steroid hormone receptors triggers a series of molecular events that ultimately affect the estrogen- or progesterone-responsive elements present in different target genes. The transcription of HPV genes, some of which code for products with transforming activity, is dependent on the regulatory elements present in the upstream regulatory region (5). One of this transcription factorbinding sites is a glucocorticoid-responsive element found in genital HPVs, but not in cutaneous HPV types (5). This element responds to both progesterones and glucocorticoids by increasing the transcription of the E6 and E7 genes of HPV 16 and 18, two of the more prevalent high-risk HPV types (5). These data suggest an interaction between HPV and steroid hormones to promote cell proliferation, eventually leading to cell transformation.
The transformation zone of the uterine cervix where more than $90 \%$ of HPV-induced lesions and cervical cancers occur is also the genital site most sensitive to estrogens (19).

Epidemiological data suggest that the use of oral contraceptives may increase the risk of cervical neoplasia (20). Taken together with the biological evidence, these data indicate that young women taking oral contraceptives are exposed to a higher risk of developing cervical neoplasias, especially if they were also exposed to high-risk HPVs. What is not clear, however, is how the regulation of the expression of steroid receptors may interfere with this process. Additional studies with a larger number of patients will be necessary to establish the relevance of the association between hormone receptors and HPV-induced lesions in the uterine cervix.

\section{Acknowledgments}

We are indebted to Dr. Eduardo L. Franco, Department of Oncology, Division of Epidemiology, McGill University, Montreal, Canada, and to Dr. Maria Mitzi Brentani, Department of Radiology, Division of Oncology, University of São Paulo, USP, Brazil, for continuous support and advice.

\section{References}

1. Schiffman MH, Bauer HM, Hoover RN et al. (1993). Epidemiologic evidence showing that human papillomavirus infection causes most cervical intraepithelial neoplasias. Journal of the National Cancer Institute, 85: 958-964.

2. Villa LL (1997). Human papillomaviruses and cervical cancer. Advances in Cancer Research, 71: 321-341.

3. Franco EL, Rohan TE \& Villa LL (1999). Epidemiologic evidence and human papillomavirus infection as a necessary cause of cervical cancer. Journal of the National Cancer Institute, 91: 506-511.

4. Chen YH, Huang LH \& Chen TM (1996). Differential effects of progestins and estrogens on long control regions of human papillomavirus types 16 and 18. Biochemical and Biophysical Research Communications, 224: 651-659.

5. Chan WK, Klock G \& Bernard HU (1989). Progesterone and glucocorticoid response elements occur in the long control regions of several human papillomaviruses involved in anogenital neoplasia.
Journal of Virology, 63: 3261-3269.

6. Mader S, Kumar V, de Verneuil H \& Chambon P (1989). Three amino acids of the oestrogen receptor are essential to its ability to distinguish an estrogen from a glucocorticoid-responsive element. Nature, 338: 271-274.

7. Picard D, Kumar V, Chambon P \& Yamamoto KR (1990). Signal transduction by steroid hormones: nuclear localization is differentially regulated in estrogen and glucocorticoid receptors. Cell Regulation, 1: 291-299.

8. Monsonego J, Magdelenat H, Catalan F, Coscas Y, Zerat L \& Sastre $X$ (1991). Estrogen and progesterone receptors in cervical human papillomavirus related lesions. International Journal of Cancer, 48 : 533-539.

9. Masood S, Rhatigan RM, Wilkinson EW, Barwick KW \& Wilson WJ (1993). Expression and prognostic significance of estrogen and progesterone receptors in adenocarcinoma of the uterine cervix. An 
immunocytochemical study. Cancer, 72: 511-518.

10. Kim JW, Sung HR, Kim DK \& Song CH (1992). Estrogen and progesterone receptor assay in carcinoma of the cervix with monoclonal antibodies. Gynecologic Oncology, 47: 306-310.

11. Robertson DI, Paslawski D, Duggan MA, Stuart GCE \& Nation JG (1993). Estrogen and progesterone receptor, human papillomavirus, and DNA ploidy analysis in invasive carcinoma of the cervix in pregnancy. American Journal of Clinical Pathology, 100: 18-21.

12. Keng S, Yueng W \& Ngan $H$ (1994). Estrogen and progesterone receptors in normal cervix and primary cervical carcinoma. Chinese Medical Journal, 107: 648-652.

13. Mosny DS, Herholz J, Degen W \& Bender HG (1989). Immunohistochemical investigation of steroid receptors in normal and neoplastic squamous epithelium of the uterine cervix. Gynecologic Oncology, 35: 373-377.

14. Taylor CR (1996). Paraffin section immunocytochemistry for estrogen receptor: the time has come. Cancer, 77: 2419-2422.

15. Bauer HM, Greer CE \& Manos MM (1992). Determination of genital human papillomavirus infection by consensus PCR applications. In: Herrington CS \& Mcgee JO (Editors), Diagnostic Molecular Pathology: A Practical Approach. Oxford University Press, Oxford, UK.
16. Bernard HU, Chan SY, Manos MM, Ong CK, Villa LL, Delius $H$, Peyton CL, Bauer HM \& Wheeler CM (1994). Identification and assessment of known and novel human papillomaviruses by polymerase chain reaction amplification, restriction fragment length polymorphisms, nucleotide sequence, and phylogenetic algorithms. Journal of Infectious Diseases, 170: 1077-1085.

17. Thompson EA (1990). Fisher's contributions to genetical statistics. Biometrics, 46: 905-914.

18. Walboomers JM, Jacobs MV, Manos MM, Bosch FX, Kummer JA, Shah KV, Snijders PJ, Peto J, Meijer CJ \& Munoz N (1999). Human papillomavirus is a necessary cause of invasive cervical cancer worldwide. Journal of Pathology, 189: 12-19.

19. Elson DA, Riley RR, Lacey A, Thordarson G, Talamantes FJ \& Arbeit JM (2000). Sensitivity of the cervical transformation zone to estrogen-induced squamous carcinogenesis. Cancer Research, 60: 12671275.

20. Moreno V, Bosch FX, Munoz N, Meijer CJ, Shah KV, Walboomers JM, Herrero R \& Franceschi S (2002). Effect of oral contraceptives on risk of cervical cancer in women with human papillomavirus infection: the IARC multicentric case-control study. Lancet, 359: 1085-1092. 